$2-2014$

\title{
Impact of Macrophage Deficiency and Depletion on Continuous Glucose Monitoring In Vivo
}

\author{
Ulrike Klueh \\ University of Connecticut School of Medicine and Dentistry \\ Yi Qiao \\ University of Connecticut School of Medicine and Dentistry \\ Jackman T. Frailey \\ University of Connecticut School of Medicine and Dentistry \\ Donald L. Kreutzer \\ University of Connecticut School of Medicine and Dentistry
}

Follow this and additional works at: https://opencommons.uconn.edu/uchcres_articles Part of the Medicine and Health Sciences Commons

\section{Recommended Citation}

Klueh, Ulrike; Qiao, Yi; Frailey, Jackman T.; and Kreutzer, Donald L., "Impact of Macrophage Deficiency and Depletion on Continuous Glucose Monitoring In Vivo" (2014). UCHC Articles - Research. 224.

https://opencommons.uconn.edu/uchcres_articles/224 


\title{
Impact of macrophage deficiency and depletion on continuous glucose monitoring in vivo
}

\author{
Ulrike Klueh $^{a, b,{ }^{,}, \text {Yi Qiao }}{ }^{a, b}$, Jackman T. Frailey ${ }^{a, b}$, and Donald L. Kreutzera,b \\ ${ }^{a}$ Center for Molecular Tissue Engineering, University of Connecticut, School of Medicine, \\ Farmington, CT 06030, USA \\ bDepartment of Surgery, University of Connecticut, School of Medicine, Farmington, CT 06030, \\ USA
}

\section{Abstract}

Although it is assumed that macrophages (MQ) have a major negative impact on continuous glucose monitoring (CGM), surprisingly there is no data in the literature to directly support or refute the role of MQ or related foreign body giant cells in the bio-fouling of glucose sensors in vivo. As such, we developed the hypothesis that MQ are key in controlling glucose sensor performance and CGM in vivo and MQ deficiencies or depletion would enhance CGM. To test this hypothesis we determined the presence/distribution of MQ at the sensor tissue interface over a 28-day time period using F4/80 antibody and immunohistochemical analysis. We also evaluated the impact of spontaneous MQ deficiency (op/op mice) and induced-transgenic MQ depletions (Diphtheria Toxin Receptor (DTR) mice) on sensor function and CGM utilizing our murine CGM system. The results of these studies demonstrated: 1) a time dependent increase in MQ accumulation (F4/80 positive cells) at the sensor tissue interface; and 2) MQ deficient mice and MQ depleted C57BL/6 mice demonstrated improved sensor performance (MARD) when compared to normal mice (C57BL/6). These studies directly demonstrate the importance of MQ in sensor function and CGM in vivo.

\section{Keywords}

Macrophages; Diabetes; Implantable glucose sensors; Continuous glucose monitoring

\section{Introduction}

The central role of monocyte related cells (MRC), i.e. macrophages (MQ), giant cells (GC) and dendritic cells (DC), as well as their products, in controlling tissue reactions associated with human disease, including foreign body reactions (FBR), is universally accepted.

Although MQ are histologically associated with implantable biosensors in vivo, there is no direct in vivo evidence of the role for MQ or their products in the loss of sensor function seen in vivo. Filling this gap in our understanding would not only clarify the role of MRC and their products in the loss of sensor function in vivo, it will also help identify key targets for therapeutic intervention and sensor design in an effort to extend sensor lifespan in vivo.

To begin to fill this gap in our knowledge we propose to determine the contribution of monocyte/macrophages (M/MQ) to sensor performance in vivo. In order to demonstrate the 
direct contributions of $\mathrm{M} / \mathrm{MQ}$ in controlling sensor function and CGM in vivo, we first characterized MQ accumulation at sensor-tissue interface at sensor implantation sites using immunohistochemical technology specific for MQ. We also evaluated CGM performance in mutant mice deficient in M/MQ (op/op mice) [1] and in mice depleted of M/MQ (Human Diphtheria Toxin Receptor (hDTR) knock-in mice) [2]. Normal littermates (op/op studies) or C57BL/6 (hDTR studies) mice were among the controls for these studies. The results of these studies demonstrated that 1) MQ accumulate at sensor-tissue interface during CGM ultimately forming a MQ barrier surrounding the implanted sensor in vivo; and 2) that either deficiency or depletion of M/MQ enhances CGM when compared to CGM in normal/control mice. Using these spontaneous and transgenic models clearly demonstrates the importance of MQ in sensor function and CGM, and underscores the need for future studies to understand and overcome negative impacts of MQ on CGM in vivo.

\section{Materials and methods}

\subsection{Mouse models}

All mice used in these studies where obtained from Jackson Laboratories, Bar Harbor Maine. These mice included op/op mice (B6; C3Fe a/a-Csf1op/J, Jax Stock \# 00231), DTR mice (B6.FVB-Tg(ITGAM-DTR/EGFP)34Lan/J, Jax Stock \# 006000) and C57BL/6J mice (Jax Stock \# 00664).

\subsection{Glucose sensors, implantation and murine continuous glucose sensor (CGM) system}

All modified Navigator glucose sensors used in these in vivo studies were obtained from Abbott Diabetes Care (Alameda California). Glucose sensors were implanted into mice and continuous glucose monitoring (CGM) was undertaken as described recently [3-5]. Blood glucose reference measurements were obtained at least daily using blood obtained from the tail vein of the mouse and a FreeStyle ${ }^{\circledR}$ Blood Glucose Monitor. The Institutional Animal Care and Use Committee of the University of Connecticut Health Center (Farmington, CT) approved all the studies involving mice.

\subsection{Glucose sensor function in macrophage deficient mice (op/op mice)}

Heterozygous op/op breeding pairs were obtained from Jackson Lab, Bar Harbor, Maine. Homozygous MQ deficient and phenotypically normal littermates (heterozygous and homozygous) (LM) were derived from the breeding pairs. The op/op macrophage deficient mice are generally greater than $85 \%$ deficient in circulating monocytes $[1,6]$. Both the op/op macrophage deficient mice and normal littermates were evaluated in the murine CGM model described above. Normal littermates of the homozygous op/op mice served as controls for the op/op studies. Using these mice, the role of CSF-1 dependent M/MQ deficiency on sensor induced tissue reactions and sensor performance in vivo was determined.

\subsection{Glucose sensor function in macrophage depleted human diphtheria toxin receptor (hDTR) chimeric mice}

The development of transgenic mice expressing the diphtheria toxin receptor driven by a CD11b promoter provides an elegant method to selectively deplete MQ in mice [2,7-9]. Due to the $\mathrm{CD} 11 \mathrm{~b}$ driven expression of the human diphtheria toxin receptor on monocytemacrophages, the addition of small intravenous dosages of diphtheria toxin to these transgenic mice triggers highly effective apoptotic destruction of all monocyte/macrophage populations for short-term studies. However for long-term macrophage depletion studies, chimeric DTR-CD11b mice are utilized to minimize toxicity and death of hDTR-CD11b mice due to long-term DT injections on resident macrophages [10]. The protocol for 
obtaining DTR-chimera mice involves injection of hDTR mouse derived bone marrow into irradiated normal LM mice (C57BL/6) (see Fig. 1). 4-8 weeks post bone marrow treatment this approach yielded a chimeric mouse with circulating DTR-monocyte/MQ (susceptible to DT) and resident tissue MQ, which are resistant to DT. Since only blood bone marrow derived monocyte/macrophages (M/MQ) are recruited to sites of sensor implantation this approach depleted circulating M/MQ systemically or locally by injection of DT. DT injection into the hDTR chimera mice generally reduces circulation monocytes to $<50 \%$ of normal levels, but DT injections have no effects on normal mice. The resulting chimeric mice and control C57BL/6 mice were evaluated in the murine CGM model described above.

\subsection{CGM data analysis for murine CGM models}

Reference blood measurements and sensor output were used to calculate the mean absolute relative difference (MARD) over a four-week experiment for the three groups of mice op/ $o p$, hDTR and control mice [11]. Equations (1.1) through (1.3) describe the MARD calculation in detail. Sensitivity $(\mathrm{S}$ in $\mathrm{mg} / \mathrm{dL} \cdot \mathrm{nAmp}$ ) is calculated for each mouse experiment based on the reference blood glucose (BG) and the sensor output (I in nA) measurements in an initial reference stage of the experiment, i.e. $k$ in Equation (1.2) is approximately 5, for the first initial 5 measurements across 2 days.

$$
\begin{gathered}
\operatorname{MARD}(\text { mean })=\left(\left(\sum_{i}^{n} \frac{\left|\mathrm{CGM}_{i}-\mathrm{BG}_{i}\right|}{\mathrm{BG}_{i}}\right) / n\right) \times 100 \% \\
\text { Sensitivity }(S)=\left(\left(\sum_{i}^{k}\left(\mathrm{BG}_{i} / I_{i}\right)\right) / k\right) \\
\mathrm{CGM}_{i}=S \times I_{i}
\end{gathered}
$$

Since most of the mean MARD values were non-normal in distribution, Kruskal-Wallis tests were used to conduct statistical comparisons among the 3 groups of MARD values, as a non-parametric equivalent to analysis of variance (ANOVA). Mann-Whitney $U$ tests were conducted to determine the statistical differences between pairs of average mean MARD values, as non-parametric equivalents to student $t$-tests. Those variables that were normally distributed were the MARD values for mice that were normal heterozygous littermates of the homozygous op/op macrophage deficient mice (MQ-LM), Chimeric with No DT injection (for all time selections), and C57BL/6 DT injected (for all time selections except week 2).

\subsection{Histopathologic analysis of tissue reactions at glucose sensor implantation sites}

In order to evaluate tissue responses to glucose sensor implantation at various time points, individual mice were euthanized and the full thickness of the skin and sensors were removed enbloc in approximately $3 \times 3 \mathrm{~cm}$ sections and immediately placed in tissue fixative. Tissue was fixed in zinc buffer for $24 \mathrm{~h}$, followed by standard processing, embedded in paraffin, and sectioned. The resulting 4-6 $\mu \mathrm{m}$ sections were then stained using standard protocols for hematoxylin/eosin stain (H/E). Histopathologic evaluation of tissue reactions at sites of sensor implantation was performed on mouse specimens obtained from 1 to 28 days postsensor implantation. The tissue samples were generally examined for signs of inflammation, including leukocyte influx, fibrosis, angiogenesis, and vessel regression. To provide an initial evaluation of the inflammatory reactions at the sensor tissue interface we utilized 
semi-quantitative evaluation scoring system from 0 to 4 . For this system the tissue reactions were scored as follows:

$0=$ no inflammation (no leukocyte infiltration present near the implanted sensor),

$1=$ trace inflammation (occasional leukocyte infiltration present near the implanted sensor),

$2=$ mild inflammation (scattered and consistent leukocyte infiltration present near the implanted sensor),

$3=$ moderate inflammation (significant leukocyte infiltration near the implanted sensor),

4 = severe inflammation (dense leukocyte infiltration near the implanted sensor).

The individual histologic sections were evaluated in a double blind fashion and the mean inflammation index was determined. Since most of the average inflammation index values were non-normal in distribution, Kruskal-Wallis tests were used to conduct statistical comparisons among the 3 groups of inflammation index values of differing genetic background. Mann-Whitney $U$ tests then were conducted to determine the statistical differences between pairs of average inflammation index values, where appropriate. Variables that were normally distributed for the inflammation index analyses were Day 7 MQ-LM, Day 28 MQ-Def, Day 14 Chimeric No DT injected, and C57BL/6 and C57BL/6 DT injected for all weeks together. Microsoft Excel for Mac 2011 (version 14.1.4) and IBM SPSS Statistics 20 (release 20.0.0) were the software packages used for the calculations and statistical analyses respectively for both the MARD and inflammation indices.

\subsection{Immunohistchemical analysis of macrophages in tissue sections using anti-F4/80 antibodies}

To confirm the observations of the presence of macrophages in tissue sections, we utilized a mouse macrophage specific antibody designated anti-mouse F4/80. Anti-mouse F4/80 (@F4/80, Invitrogen Catalog \#A14800) was validated using mouse spleen tissue and standard immunohistochemical (IHC) techniques.

\section{Results}

\subsection{Validation of specificity of macrophage reactive anti-F4/80 antibody used for immunohistochemical staining of tissue}

Utilizing standard immunohistochemical techniques we first validated the specificity of the anti-F4/80 MQ staining antibody (@F4/80) on mouse spleen. As expected @ F4/80 stained macrophage containing red pulp in the spleen (positive stain indicated by reddish-brown staining of cells) but not lymphocyte containing white pulp (clear/white appearance of cells and tissue) (Fig. 2). Non-immune (normal) immunoglobulin (IgG) was used as a control and as shown in Fig. 2 did not stain any of the cells in the spleen tissue indicating specificity of the @F4/80 staining (Fig. 2). A Lower power magnification is presented in the top row \& high power magnification bottom row of Fig. 2 .

\subsection{Characterization tissue reactions and presence of $M Q$ at tissue implantation sites of glucose sensors}

To characterize the nature and distribution of MQ recruited to sites of ADC Navigator sensor in our mouse model, tissue-sensors complex were removed in-block from sites of sensor implantation, fixed and processed for histopathology at various days post implantation. H\&E results of these studies are present in Fig. 3a. The star in the Fig. 3a represents the position of the sensor within the tissue. The results of these studies indicated 
that at 1-day post implantation, the tissue reactions at the site of sensor implantation was not only characterized by a mixture of inflammatory cells, mainly poly-morphonuclear (PMN), but also some mononuclear leukocytes. At day 3, the inflammatory process had evolved to predominately mononuclear leukocytes, which seen accumulating at the sensor tissue interface. The accumulation of mononuclear leukocytes at the sensor-tissue interface continued at day 7 and 14 post-sensor implantation. Sparse numbers of PMN were seen at the peripheral edges of the inflammatory reactions on days 3-14. Fibroblasts and collagen deposition was seen on days 7 and 14 (Mason trichrome staining, data not shown). These observations are consistent with the foreign body reactions induced by biomaterials and implants $[12,13]$.

To confirm that mononuclear cells in the H\&E study (Fig. 3a) were truly macrophages, we next characterized the presence and distribution of macrophages at sites of sensor implantation using standard IHC (@F4/80 antibodies). These studies detected scattered MQ as early as 1-day post senor implantation (Fig. 3b). MQ continued to accumulate within the tissue adjacent to the implanted sensor over the next 14 days (Fig. 3). Accumulation of MQ at the sensor-tissue interface continued to increase, ultimately forming a large MQ "band/ barrier" surrounding the sensor at day 14-post implantation and beyond. As a control normal Ig was tested at all time points and was negative for all time points. As a representative example of the normal IgG staining for Day 1 and 14-post sensor implantation are presented in Fig. 3b.

\subsection{Continuous glucose monitoring in macrophage deficient (op/op) mice}

Macrophage deficient mice (homozygous op/op mice), which are genetically deficient in $\mathrm{M} /$ MQ because of a CSF-1 deficiency, have been used to determine the role of monocyte/ macrophages in a variety of murine disease models $[14,15]$. Using these mice we undertook studies to determine the role of M/MQ deficiency on sensor function in vivo. For controls sensor function in normal heterozygous littermates of the homozygous op/op macrophage deficient mice (MQ-LM) were evaluated. CGM was evaluated for up to 28 days post-sensor implantation (DPSI). For these studies we evaluated the effect of macrophages on sensor function by measuring sensor output (nA) and calculating the mean absolute relative difference (MARD) between blood glucose measurements from implanted glucose sensors and standard clinical glucometers over a four-week experiment for MQ deficient $o p / o p$ (MQ-Def) mice and control/normal littermates (MQ-LM) and (Fig. 4). Fig. 4 demonstrates the effect of macrophage genetic background on separate cumulative weekly mean MARD over the 4-week experiment. Statistical analysis of this data demonstrated that the MQ-Def and MQ-LM mice have significantly enhanced total mean MARD (i.e. all data over the entire 4 week experiment), $p=0.0471$. This $p$-value was calculated by Mann-Whitney $U$ test, as non-parametric equivalent to a student $t$-test, because only the MQ-LM group had normally distributed mean MARD values. The sample sizes are relatively large for such investigations with approximately 20 or more mice in each group $(26,21$, and 18 mice in the C57BL/6, MQ-LM, and MQ-Def groups respectively). These studies demonstrated better long-term (>2 week post-sensor implantation) sensor function in MQ deficient mice when compared to normal littermate controls.

In addition to evaluating sensor function we evaluated the effect of MQ deficiency on sensor induced tissue reactivity over the four-week experiment for MQ deficient (MQ-Def, op/op) and MQ-LM (normal littermates) mice (Fig. 5). Fig. 5 demonstrates the trends in average inflammation indices from no inflammation at time 0 to week 4 , distinguished by genetic background. The MQ-Def mice have significantly less inflammation in weeks 1 and 2 than their control normal littermates (MQ-LM), but have statistically approximately the same index of inflammation in weeks 3 and 4. In interpreting this data it is important to note that 
the op/op mice are deficient in monocyte/macrophages (M/MQ) not devoid of M/MQ and general have $10-20 \%$ of normal circulating monocytes $[1,6]$. It is also important to note that the tissue reactions at sensor implantation sites involve only a few mm of tissue so only a limited number of MQ are in need to be recruited to impact CGM. Additionally it is known that foreign body reactions occur in op/op mice but are less intense compared to normal mice, e.g. smaller giant cell formation [15]. Thus, we believe this M/MQ deficiency is what accounted for the limited inflammation seen in the first 2 weeks of the op/op MQ-Def mice, but given enough time eventually the recruited MQ accumulate at the sensor-tissue interface that equals the MQ accumulation seen in the MQ-LM group. To confirm and extend these observations we next determined the impact of MQ depletion on CGM, we undertook studies using transgenic mice whose MQ can be deleted using injections of diphtheria toxin[2,7-9].

\subsection{Continuous glucose monitoring in macrophage depleted diphtheria toxin receptor (DTR) chimeric mice}

Transgenic mice that express the human diphtheria toxin receptor driven by a CD11b promoter provides an elegant method to selectively deplete MQ in mice [2,7-9]. Due to this expression of diphtheria toxin receptors on monocyte-macrophages the addition of small intravenous dosages of diphtheria toxin to these transgenic mice results in destruction of all monocyte/macrophage populations in the mice in short term studies. However for long-term macrophage depletion studies, chimeric DTR-CD11b mice are utilized to minimize toxicity and death of hDTR-CD11b mice due to long-term DT injections on resident macrophages [10]. Initial studies showed that DT mediated M/MQ depletion could be maintained for up to 3 weeks before toxicity was seen (data not presented). CGM was compared in normal C57BL/6 mice \pm DT injections, as well as chimera DTR mice \pm DT injection. The DT induced monocyte depletion in the chimera DTR mice significantly enhanced CGM when compared to C57BL/6 \pm DT injections or DTR mice without DT injections (Fig. 6).

Statistically significant differences were observed for the average MARD for all DT injected chimeric mice but not for any of the control mice (Table 1). There is no statistically significant difference between the cumulative MARD values for the C57BL/6 control mice or the chimeric mice that did not receive DT treatment (Table 1). These $p$-values were calculated by Mann-Whitney $U$ tests, as non-parametric equivalents to student $t$-tests, because only the Chimeric No DT injected and C57BL/6 DT injected treatment groups had normally distributed mean MARD values. These results support our prior studies utilizing op/op mouse (MQ deficient mouse) and support the key role of M/MQ in controlling CGM in vivo.

Using results from the chimeric mouse studies, we also evaluated the effect of macrophages on inflammation over a three-week experiment for chimeric mice treated with buffer (Ch) or diphtheria toxin $(\mathrm{Ch}+\mathrm{DT})$, as well as normal C57BL/6 mice treated with buffer (C57BL/6) or diphtheria toxin $(\mathrm{C} 57 \mathrm{BL} / 6+\mathrm{DT})$. Fig. 7 demonstrates the trends in average inflammation indices in the chimeric mouse studies from no inflammation at time 0 to week 3 ,

distinguished by treatment. The chimeric mice treated with diphtheria toxin $(\mathrm{Ch}+\mathrm{DT})$ have consistently significantly less inflammation than all the other controls, throughout the three week experiment. These results confirm the trends in MARD in which chimeric mice treated with diphtheria toxin $(\mathrm{Ch}+\mathrm{DT})$ have consistently significantly lower MARD than their controls, throughout the three-week experiment.

\section{Discussion}

MQ are known to be important cells in host defense against foreign objects, including microorganisms, via metabolically intense activities (i.e. glucose metabolism) such as 
chemotaxis, phagocytosis and generation of reactive oxygen species [16,17]. Sensors, like microorganisms, are foreign objects and also trigger these same intense metabolic activities. Although it is assumed that these MQ have a negative impact on CGM, currently there is no data in the literature to support or refute this assumption. To help fill this critical gap in the literature we utilized a murine model for CGM and mice that are genetically deficient in MQ to determine the impact of the deficiency on sensor performance in vivo. We believe that our present studies demonstrate the critical role of MQ in the loss of sensor function in vivo.

Recent advances in our understanding of the role and regulation of MQ and lymphocytes in human diseases such as cancer, heart disease, arthritis, and skin diseases (e.g. psoriasis) have come from the utilization of the mouse. The mouse has been used to develop basic understandings of the pathogenesis of these diseases and develop effective approaches and drugs for treating them. Unfortunately the power of the mouse, with its massive literature, genetics, wide array of spontaneous and transgenic mice as well as reagents (antibodies, recombinant products, genes etc.) has not been used to advance the understanding or control tissue responses surrounding glucose sensors or any other implantable device.

\subsection{Impact of macrophage deficiency and depletion on continuous glucose monitoring in vivo}

It is known that colony-stimulating factor-1 (CSF-1) drives the bulk of development of monocytes (M) in vivo [1]. Macrophage deficient mice (homozygous op/op mice) are genetically deficient in M/MQ due to a mutation in the colony-stimulating factor-1 (CSF-1) gene. This absence of biologically active CSF-1 results in major monocytopenia [18-20]. Additional studies have demonstrated that $o p / o p$ mice have diminished responses in various murine models $[14,15]$. Interestingly op/op mice have been shown to display diminished form of foreign body reactions [15]. Using these mice we determined the role of CSF-1 dependent M/MQ deficiency on controlling sensor induced tissue reactions and function in vivo. Although the results of these studies showed better overall CGM performance for the op/op mice, when compared to their normal littermates they where not as dramatic as we thought they would be. We believe this is the result of the fact that the op/op mice are only deficient in circulating monocytes not devoid of them. As such, over the 4 week test period some monocytes were recruited to the sensor implantation sites and were converted to MQ. This compromised sensor performance as MQ accumulated at the sensor-tissue interface over the 28 day studies. It is also important to note that the tissue reactions at sensor implantation sites involve only a few $\mathrm{mm}$ of tissue. Thus we believe this M/MQ deficiency is what accounted for the limited inflammation seen in the first 2 weeks of the MQ-Def mice, but given enough time eventually the recruited MQ accumulate at the sensor-tissue interface that equals the MQ accumulation seen in the MQ-LM group. To confirm and extend these observations we next determined the impact of MQ depletion on CGM using transgenic mice who's MQ can be deleted using injections of diphtheria toxin [2,7-9].

\subsection{Glucose sensor function in macrophage depleted diphtheria toxin receptor (DTR) chimeric mice}

Chemical/drug based depletion of M/MQ is a simple classic approach to defining the role of MQ in disease states. However, as is the case with all chemical/drug treatments, there can always be concerns of possible side effects/artifacts. The development of transgenic mice that express the diphtheria toxin receptor driven by a CD11b promoter provides an elegant method to selectively deplete MQ in mice [2,7-9]. In this transgenic mouse, the diphtheria toxin receptor is only over-expressed in cells that express CD11b, i.e. monocytes/ macrophages. Because of this CD11b driven expression of the diphtheria toxin receptor on monocyte-macrophages, the addition of small i.v. doses of diphtheria toxin to these transgenic mice, triggers highly effective apoptotic destruction of all monocyte/macrophage 
populations in the mice [2]. Generally, these mice have been used effectively to investigate the role of MQ in short term studies (days to one week), but because of eventual toxicity to resident MQ and death of the hDTR mice have more limited value. To overcome this problem usually chimera mice obtained by injection of bone marrow from hDTR into irradiated control mice are used [2]. For our present studies we utilized these hDTR chimeric mice to extend the M/MQ depletion time-frame to 3 weeks. Using the hDTR chimeric mice we found that DT depletion of MQ dramatically decreased inflammation at the site of sensor implantation (Fig. 7) as well as enhanced CGM when compared to the various control mice \pm DT injection (Fig. 6). We believe this chimera hDTR data both confirms and extends the op/op data presented earlier and supports our hypothesis on the importance of MQ in controlling both tissue reactions and glucose sensor function at sites of sensor implantation in vivo. Future studies to dissect the role of foreign body giant cells as well as MQ in controlling tissue reactions and sensor performance will be in need to expand our understanding of the role(s) of monocyte related cells in controlling CGM in vivo, as well as developing rationale approaches to overcoming negative impacts of MQ and the tissue reactions they control, to achieve enhanced glucose sensor accuracy and lifespan in vivo.

\subsection{Hypothetical model of the role of monocytes, macrophage and related cells in sensor- induced tissue reactions and sensor function in vivo}

Based on the present data as well as the literature related to the role of sensor induced tissue reactions and CGM we have developed the following hypothetical model of the role on monocyte related cells in controlling tissue reactions and sensor function in vivo (Fig. 8). We hypothesize that monocyte related cells such as macrophages and foreign body giant cells (GC) play a central role in both direct bio-fouling and indirect bio-fouling of glucose sensors in vivo. MQ and GC can directly bio-foul glucose sensors by creating cellular barriers that surround the implanted sensor. This effect can slow glucose diffusion to the implanted sensor site as well as metabolize glucose and oxygen thereby creating artificially low glucose levels and sensor readings relative to the general interstitial glucose levels that exist distant from the implanted sensor. Due to the close proximity of this layer of monocyte related cells to the implanted sensor, these cells can also produce relatively high concentrations of agents that can damage the glucose sensor such as oxygen radicals. In addition to the direct bio-fouling of the sensor by monocyte related cells these cells can produce extremely potent inflammatory mediators that promote additional monocyte recruitment from the vasculature via the vascular endothelial cells (VEC) as well as activate monocytes into specific macrophage subpopulations (e.g. M1 or M2 macrophages). M1 macrophages can further amplify the inflammatory reactions through expression of proinflammatory mediators including cytokines. M2 macrophages can stimulate wound-healing processes including angiogenesis and fibrosis. Although angiogenesis can be beneficial to sensor function it also promotes fibroblast proliferation and collagen synthesis resulting in fibrosis. As fibrosis increases at the sensor implantation site it induces blood vessel regression as well as slowing glucose diffusion toward the sensor. In summary, we believe that monocytes and monocyte related cells such as macrophages and foreign body giant cells are key targets for the therapeutic intervention to control tissue reactions that bio-foul glucose sensors. Additionally understanding the specific cell sensor interactions that occur at the tissue sensor interface should provide a more rationale approach to designing future generation of glucose sensors used in CGM.

\section{Conclusion}

Implantable glucose sensors used in Continuous Glucose Monitoring (CGM) of patients with diabetes are known to induce foreign body reactions characterized by accumulation of macrophages (MQ) at the sensor-tissue interface. Although it is assumed that these MQ 
have a negative impact on CGM, currently there is no data in the literature to support or refute this assumption. To fill this gap of our knowledge we utilized our mouse model of continuous blood glucose monitoring as well as macrophage deficient mice to determine the impact of this deficiency on sensor function in vivo. CGM in all mice was evaluated for up to 28 days post-sensor implantation (PSI) for MQ deficient mice (op/op), MQ depleted mice (chimera mice) and control mice (C57BL/6j). We also characterized MQ presence and distribution at the sensor tissue interface using anti-MQ specific antibody (F4/80) and standard immunohistochemical (IHC) techniques. Our immunohistological studies demonstrate an increased number of MQ accumulations at site of sensor implantation. The increased appearance of MQ was also associated with decreased sensor functionality in vivo. MQ deficient and depleted mice showed much better sensor functionality i.e. improved CGM.

\section{Acknowledgments}

We would like to thank Omar Antar for his skillful assistance in the statistical analyses. The National Institute of Diabetes and Digestive Kidney Diseases provided support for these studies.

\section{References}

[1]. Wiktor-Jedrzejczak WW, Ahmed A, Szczylik C, Skelly RR. Hematological characterization of congenital osteopetrosis in op/op mouse. Possible mechanism for abnormal macrophage differentiation. J Exp Med. 1982; 156(5):1516-27. [PubMed: 7130905]

[2]. Duffield JS, Forbes SJ, Constandinou CM, Clay S, Partolina M, Vuthoori S, et al. Selective depletion of macrophages reveals distinct, opposing roles during liver injury and repair. J Clin Invest. 2005; 115(1):56-65. [PubMed: 15630444]

[3]. Klueh U, Liu Z, Cho B, Ouyang T, Feldman B, Henning TP, et al. Continuous glucose monitoring in normal mice and mice with prediabetes and diabetes. Diabetes Technol Ther. 2006; 8(3):40212. [PubMed: 16800762]

[4]. Klueh U, Liu Z, Feldman B, Henning TP, Cho B, Ouyang T, et al. Metabolic biofouling of glucose sensors in vivo: role of tissue microhemorrhages. J Diabetes Sci Technol. 2011; 5(3):583-95. [PubMed: 21722574]

[5]. Klueh U, Liu Z, Feldman B, Kreutzer DL. Importance of interleukin 1/inter-leukin 1 receptor antagonist in short term glucose sensor function in vivo. Diabetes Sci Technol. 2010; 4(5):107386.

[6]. Naito M, Hayashi S, Yoshida H, Nishikawa S, Shultz LD, Takahashi K. Abnormal differentiation of tissue macrophage populations in 'osteopetrosis' (op) mice defective in the production of macrophage colony-stimulating factor. Am J Pathol. 1991; 139(3):657-67. [PubMed: 1887865]

[7]. Cailhier JF, Partolina M, Vuthoori S, Wu S, Ko K, Watson S, et al. Conditional macrophage ablation demonstrates that resident macrophages initiate acute peritoneal inflammation. J Immunol. 2005; 174(4):2336-42. [PubMed: 15699170]

[8]. Cailhier JF, Sawatzky DA, Kipari T, Houlberg K, Walbaum D, Watson S, et al. Resident pleural macrophages are key orchestrators of neutrophil recruitment in pleural inflammation. Am J Respir Crit Care Med. 2006; 173(5):540-7. [PubMed: 16357332]

[9]. Duffield JS, Tipping PG, Kipari T, Cailhier JF, Clay S, Lang R, et al. Conditional ablation of macrophages halts progression of crescentic glomerulonephritis. Am J Pathol. 2005; 167(5): 1207-19. [PubMed: 16251406]

[10]. Stoneman V, Braganza D, Figg N, Mercer J, Lang R, Goddard M, et al. Monocyte/macrophage suppression in CD11b diphtheria toxin receptor transgenic mice differentially affects atherogenesis and established plaques. Circ Res. 2007; 100(6):884-93. [PubMed: 17322176]

[11]. Obermaier K, Schmelzeisen-Redeker G, Schoemaker M, Klotzer HM, Kirchsteiger H, Eikmeier $\mathrm{H}$, et al. Performance evaluations of continuous glucose monitoring systems: precision absolute relative deviation is part of the assessment. J Diabetes Sci Technol. 2013; 7(4):824-32. [PubMed: 23911163] 
[12]. Anderson JM, Rodriguez A, Chang DT. Foreign body reaction to biomaterials. Semin Immunol. 2008; 20(2):86-100. [PubMed: 18162407]

[13]. Luttikhuizen DT, Harmsen MC, Van Luyn MJ. Cellular and molecular dynamics in the foreign body reaction. Tissue Eng. 2006; 12(7):1955-70. [PubMed: 16889525]

[14]. Wiktor-Jedrzejczak W, Sell KW, Szwech P, Ahmed-Ansari A. Absence of CSF-1 in macrophage-deficient op/op mice has differential effects on macrophage colony stimulating activity (M-CSA) released by various organs and cells. Folia Histochem Cytobiol. 1995; 33(4): 259-65. [PubMed: 8851055]

[15]. Iizuka T, Kohgo T, Marks SC Jr. Foreign body giant cell induction in the CSF-1-deficient osteopetrotic (op/op) mouse. Tissue Cell. 2002; 34(2):103-8. [PubMed: 12165245]

[16]. Wynn TA, Chawla A, Pollard JW. Macrophage biology in development, homeostasis and disease. Nature. 2013; 496(7446):445-55. [PubMed: 23619691]

[17]. Mantovani A, Biswas SK, Galdiero MR, Sica A, Locati M. Macrophage plasticity and polarization in tissue repair and remodelling. J Pathol. 2013; 229(2):176-85. [PubMed: 23096265]

[18]. Collins SJ, Ulmer J, Purton LE, Darlington G. Multipotent hematopoietic cell lines derived from C/EBPalpha(-/-) knockout mice display granulocyte macrophage-colony-stimulating factor, granulocyte- colony-stimulating factor, and retinoic acid-induced granulocytic differentiation. Blood. 2001; 98(8):2382-8. [PubMed: 11588034]

[19]. Naito M, Umeda S, Takahashi K, Shultz LD. Macrophage differentiation and granulomatous inflammation in osteopetrotic mice (op/op) defective in the production of CSF-1. Mol Reprod Dev. 1997; 46(1):85-91. [PubMed: 8981368]

[20]. Wiktor-Jedrzejczak W, Bartocci A, Ferrante AW Jr, Ahmed-Ansari A, Sell KW, Pollard JW, et al. Total absence of colony-stimulating factor 1 in the macrophage- deficient osteopetrotic (op/ op) mouse. Proc Natl Acad Sci U S A. 1990; 87(12):4828-32. [PubMed: 2191302] 


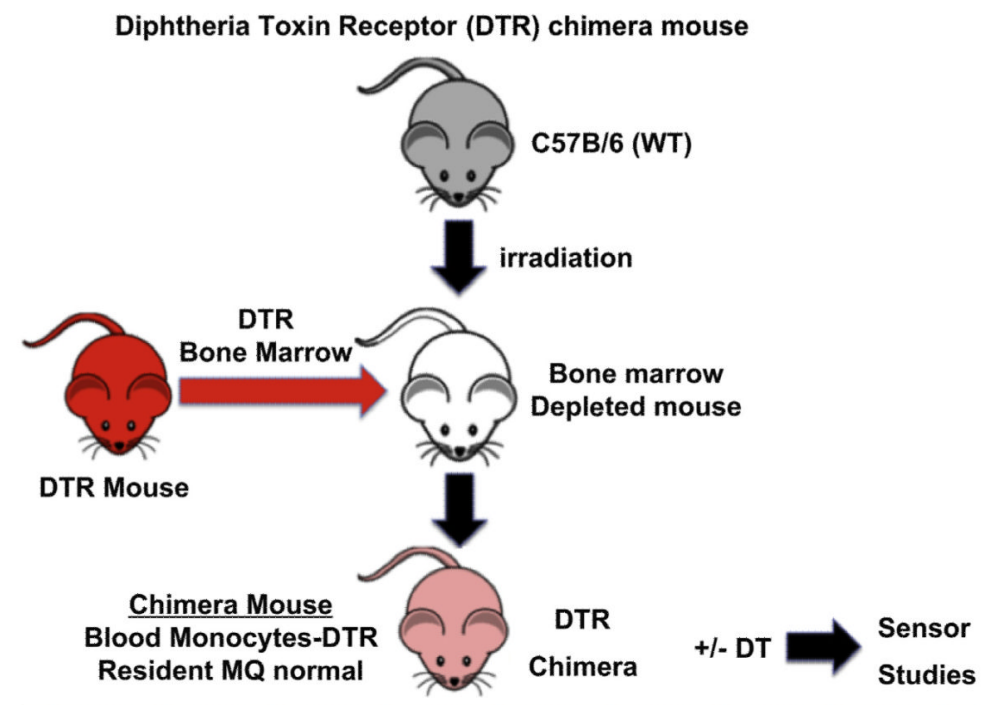

Fig. 1.

Diagram of protocol used to obtain DTR-chimera mice. Fig. 1 represents the protocol for the formation of diphtheria toxin receptor chimeric mice used for the CGM studies presented in this application. 


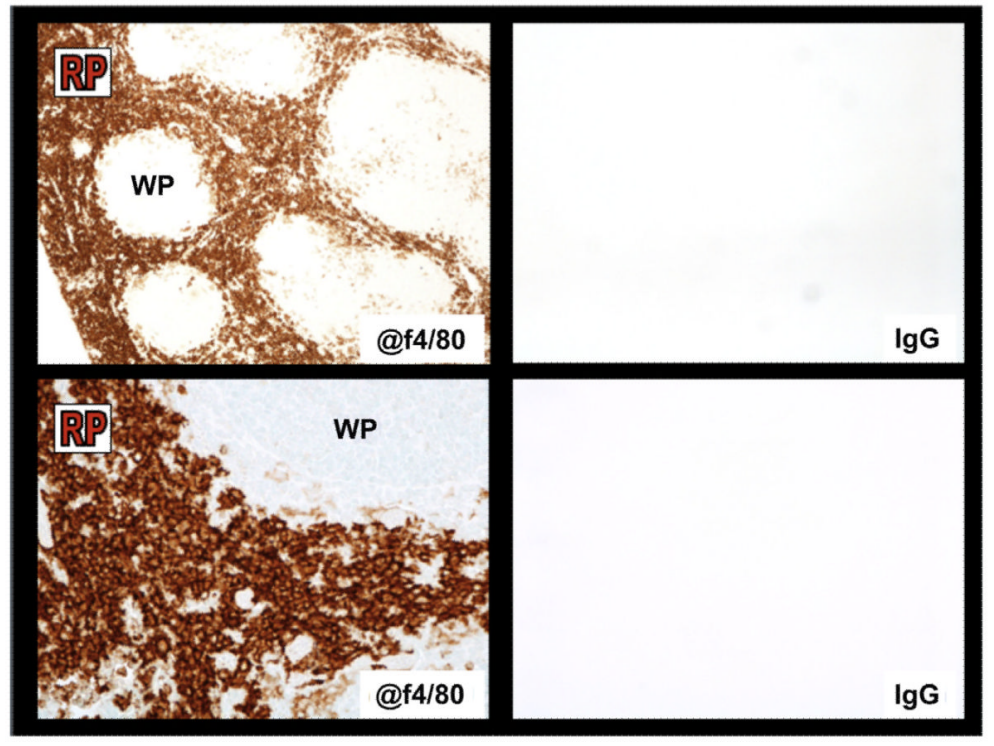

Fig. 2.

Validation of macrophage reactive antibodies (anti-F4/80) in mouse spleen. To confirm the observations of the presence of macrophages in tissue sections, we utilized a mouse macrophage specific antibody designated anti-mouse F4/80. Anti-mouse F4/80 (@F4/80) was validated using mouse spleen tissue and standard immunohistochemical (IHC) techniques. Non-immune immunoglobulin (Ig) was used as a control for non-specific binding to the spleen tissue including the lymphocyte white pulp (WP) and MQ containing red pulp (RP). @F4/80 reactive tissue-cells appear reddish-brown (i.e. MQ containing RP). As expected no@F4/80 reactivity staining was seen in the lymphocyte containing WP tissue. Non-immune Ig did not react with RP or WP. (For interpretation of the references to color in this figure legend, the reader is referred to the web version of this article.) 
a

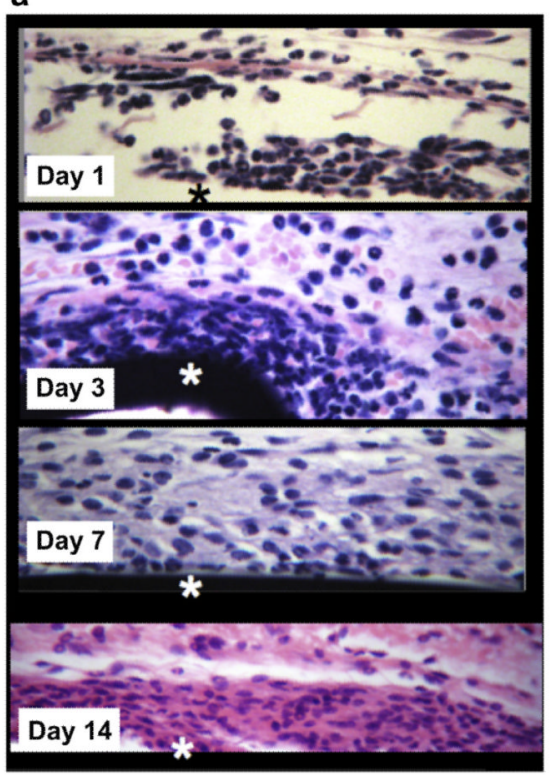

b

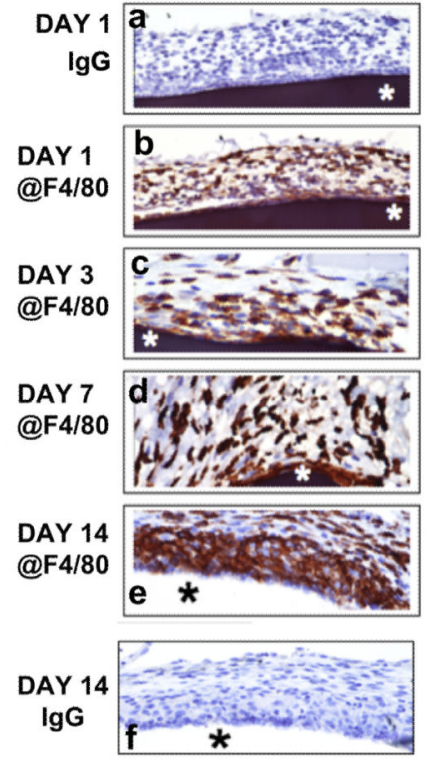

Fig. 3.

Characterization of glucose sensor induced tissue reactions at sensor implantation sites in vivo. Fig. 3 are photomicrographs of standard H\&E and @F4/80 IHC staining of glucose sensor-tissue interface at glucose sensor implantation sites in a murine model of CGM. Fig. 3a. Tissue reactions to ADC Navigator sensors (H\&E). Fig. 3a are photomicrographs of standard H\&E staining of glucose sensor-tissue interface at glucose sensor implantation sites in a murine model of CGM over 1-14 days post-sensor implantation. Fig. 3b. Characterization of the presence of F4/80 positive MQ at Tissue-Sensor Interface, Fig. 3b are photomicrographs of standard IHC staining of glucose sensor-tissue interface at glucose sensor implantation sites in a murine model of CGM using @F4/80 immunoglobulins or non-immune immunoglobulin (Ig). The tissue used for these studies were from days 1, 3, 7 and 14 days days post-sensor implantation. The star designates the sensor location within tissue. 


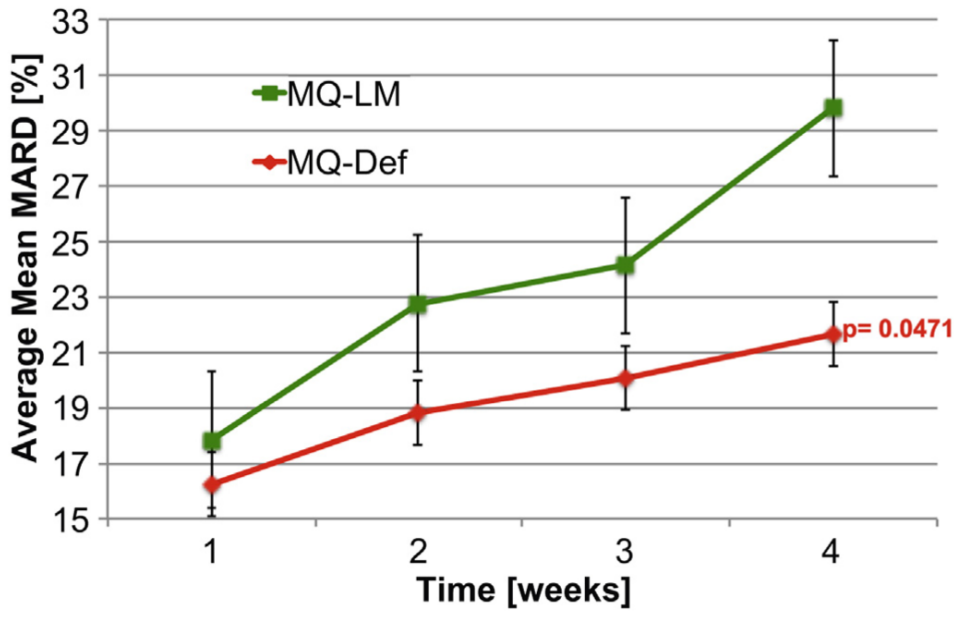

Fig. 4.

MARD trend analysis of CGM for macrophage deficient op/op mice (MQ-Def) and normal littermates (MQ-LM). Fig. 4 represents the trends in average mean MARD from weeks 1-4, by genetic background for MQ-Deficient (op/op) vs MQ-LM (normal littermates) mice. The error bars around each data point represent the standard error of the mean MARD for the particular time point and genetic background. The $p$-value indicated in red on the right side of the graph was calculated to determine the difference between total mean MARD (i.e. all data over the entire 4 week experiment) of MQ-Def and MQ-LM, by Mann-Whitney $U$ test, as non-parametric equivalent to a student $t$-test. Only the MQ-LM group had normally distributed mean MARD values. 


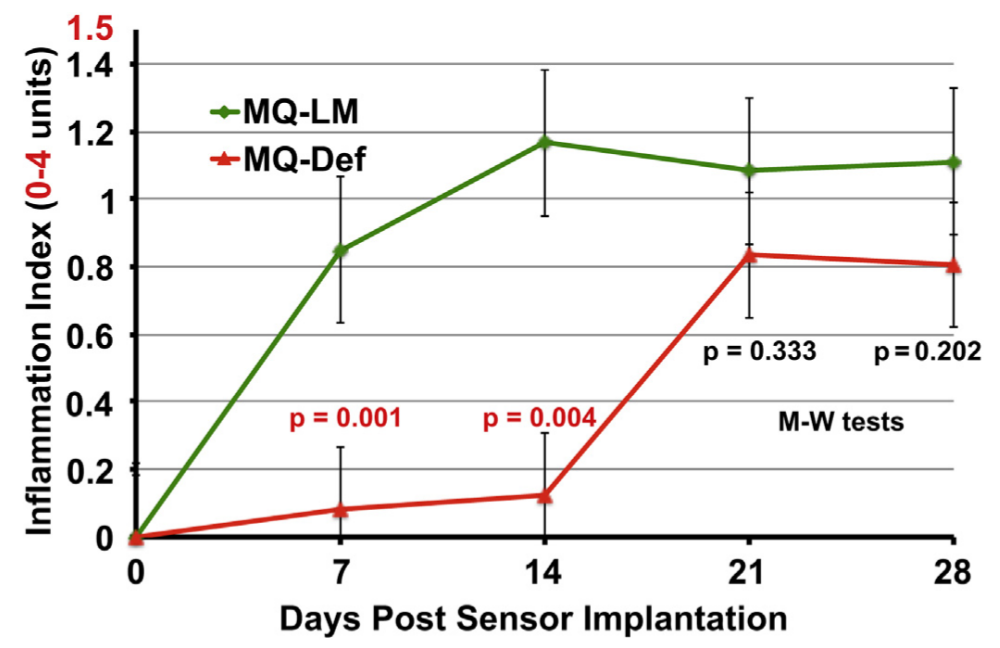

Fig. 5.

Inflammation index analysis of macrophage deficient op/op mice (MQ-Def) and normal littermates (MQ-LM). Fig. 5 represents the trends in average inflammation indices from weeks 1-4, by genetic background for MQ-Def (op/op) vs MQ-LM (normal littermates) mice. Inflammation indices range from a minimum of 0 (no inflammation) to a maximum of 4 (severe inflammation). $p$-values at the bottom represent the significance of the difference between the two groups in average inflammation index value for each individual week, by Mann-Whitney $U$ test, as non-parametric equivalent to a student t-test. The error bars around each data point represent the standard error of the average inflammation index value for the particular time point and genetic background. 


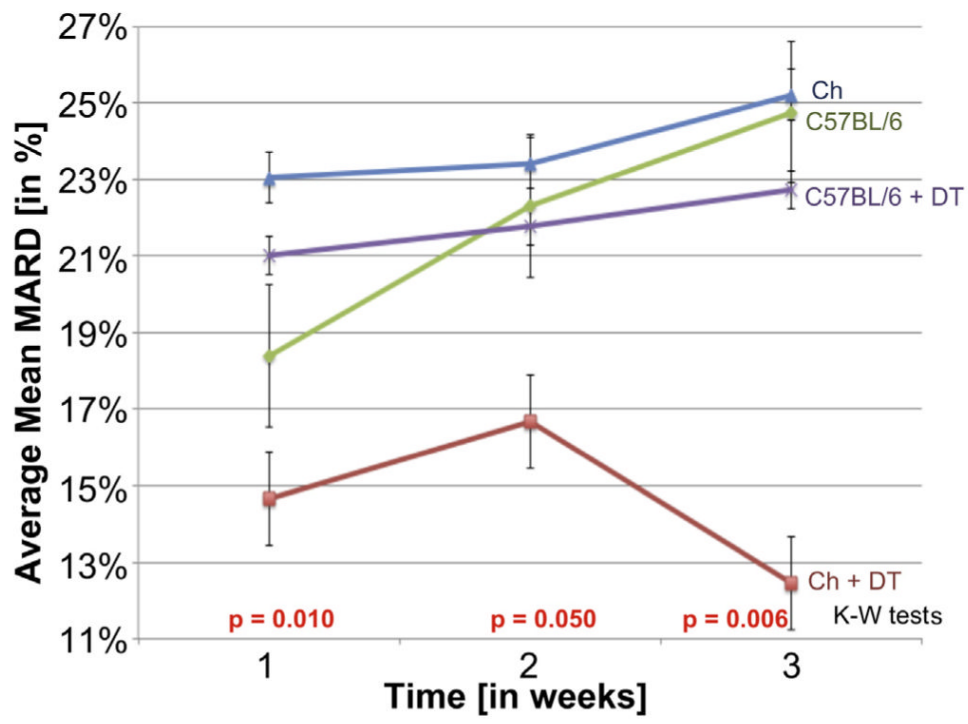

Fig. 6.

MARD trend analysis of CGM by week for diphtheria toxin (DT) receptor (DTR) chimeric mouse study. Fig. 6 represents the trends in average mean MARD from weeks 1-3, by treatment group: Chimeric mice treated with buffer $(\mathrm{Ch})$ or diphtheria toxin $(\mathrm{Ch}+\mathrm{DT})$, as well as normal C57BL/6 mice treated with buffer (C57BL/6) or diphtheria toxin (C57BL/6 + DT). $p$-values at the bottom represent the significance of the difference among the four treatment groups in average mean MARD value for each individual week, by the KruskalWallis test (as non-parametric equivalent to ANOVA). The error bars around each data point represent the standard error of the mean MARD for the particular time point and treatment group. 


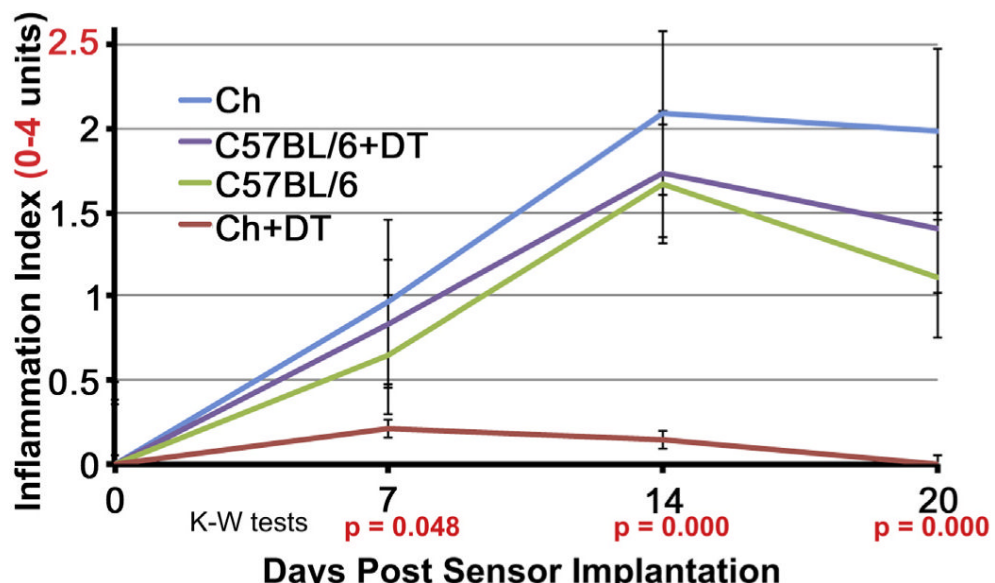

Fig. 7.

Inflammation index analysis of diphtheria toxin (DT) receptor (DTR) chimeric mouse study. Fig. 7 represents the trends in average inflammation index value from weeks $1-3$, by treatment group: Chimeric mice treated with buffer $(\mathrm{Ch})$ or diphtheria toxin $(\mathrm{Ch}+\mathrm{DT})$, as well as normal C57BL/6 mice treated with buffer (C57BL/6) or diphtheria toxin (C57BL/6 + DT). Inflammation indices range from a minimum of 0 (no inflammation) to a maximum of 4 (severe inflammation). $p$-values at the bottom represent the significance of the difference among the four treatment groups in average inflammation index value for each individual week, by the Kruskal-Wallis test (as non-parametric equivalent to ANOVA). The error bars around each data point represent the standard error of the average inflammation index value for the particular time point and treatment group. 


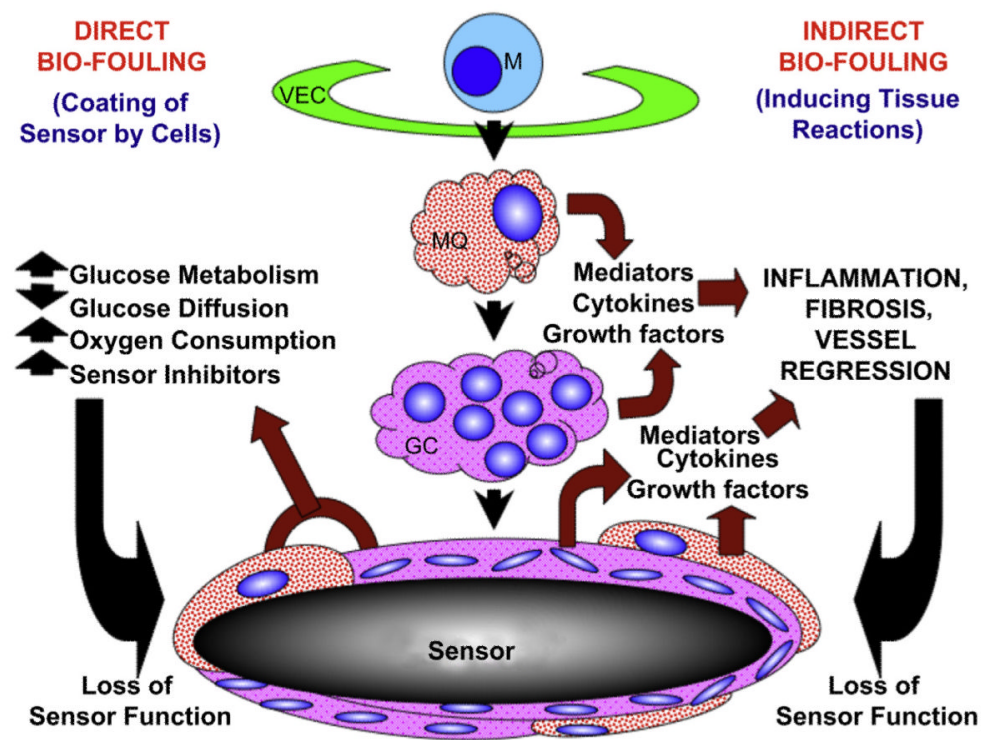

Fig. 8.

Hypothetical model of the role of monocyte, macrophage and related cells in sensor-induced tissue reactions and sensor function in vivo. Fig. 8 represents a hypothetical model of the role of monocyte related cells in sensor-induced tissue reactions and sensor function in vivo. For this model it is hypothesized that monocyte (M) related cells such as macrophages (MQ) and foreign body giant cells (GC) play a central role in both direct bio-fouling and indirect bio-fouling of glucose sensors in vivo. These monocyte related cells which are recruited from the vasculature, via vascular endothelial cells (VECs) and accumulate as a " metabolic barrier" at the tissue-sensor interface, as well as elaborate pro-inflammatory and pro-fibrotic mediators and cytokines that amplify tissue reactions at the sensor implantation site limit sensor function and CGM. 


\section{Table 1}

Average mean absolute relative difference (MARD) values at three weeks for DTR chimeric mouse studies.

\begin{tabular}{|c|c|c|c|c|}
\hline $\begin{array}{l}\text { Cumulative } 3 \text { weeks mean MARD data Mann- } \\
\text { Whitney } \\
U \text { tests }\end{array}$ & $\begin{array}{l}\text { C57BL/6 } \\
\text { average mean } \\
\text { MARD = 24.76 } \\
\pm \\
11.27 \% n=20\end{array}$ & $\begin{array}{l}\text { C57BL/6 DT Inj } \\
\text { average } \\
\text { mean MARD = } \\
22.71 \pm \\
7.88 \% n=7\end{array}$ & $\begin{array}{l}\text { Ch No DT } \\
\text { injection average } \\
\text { mean MARD = } \\
25.22 \pm \\
8.70 \% n=12\end{array}$ & $\begin{array}{l}\text { Ch DT injected } \\
\text { average } \\
\text { mean MARD = } \\
12.46 \pm \\
2.97 \% n=6\end{array}$ \\
\hline $\begin{array}{l}\text { C57BL } / 6 \text { average mean MARD }=24.76 \pm 11.27 \% n= \\
20\end{array}$ & - & 1.0000 & 0.8032 & 0.0003 \\
\hline $\begin{array}{l}\text { C57BL/6 DT Inj average mean MARD }=22.71 \pm \\
7.88 \% n=7\end{array}$ & & - & 0.5391 & 0.0140 \\
\hline $\begin{array}{l}\text { Ch No DT Injection average mean MARD }=25.22 \pm \\
8.70 \% \\
\quad n=12\end{array}$ & & & - & 0.0004 \\
\hline $\begin{array}{l}\text { Ch DT Injected average mean MARD }=12.46 \pm \\
2.97 \% n=6\end{array}$ & & & & - \\
\hline
\end{tabular}

Table 1. Presents the Average Mean Absolute Relative Difference (MARD) values at three weeks for DTR Chimeric Mouse Studies. Error values following the \pm are standard deviations from the average of the individual treatment group's MARD values. $p$-values within the boxes represent the statistical significance of the comparisons of the two treatment groups indicated in the axes, calculated by Mann-Whitney $U$ tests, as non-

parametric equivalents to student $t$-tests, where appropriate. Mann-Whitney $U$ tests were conducted because only the Chimeric No DT injected and C57BL/6 DT injected treatment groups had normally distributed mean MARD values. 